\title{
MUSIC AS A TREATMENT FOR BORDERLINE PERSONALITY DISORDER SUFFERERS WHO HAVE DEVELOPED CARDIOMETABOLIC SYNDROME
}

\author{
Esther Hunter \\ Southern Cross University (Australia)
}

\begin{abstract}
Research demonstrating the ability of music to reach the older parts of the brain responsible for emotional processing make a case for utilising specific musical compositions to deliver treatment to people with Borderline Personality Disorder. BPD has been linked to an increased risk of Cardiometabolic Syndrome (CMS), as traumatic experiences in childhood predict adverse mental and physical health in adulthood including Personality Disorders. BPD sufferers who develop CMS as a result of impulsive lifestyle choices may have their recovery inhibited by the effects of CMS. Dieting may be particularly difficult for people with BPD as food serves as a way to soothe emotional pain and depression. Emotional pain leads to making choices which increase the chances of developing health conditions which research has shown negatively affect mood and memory function. Remission of BPD requires maintaining a reduction in impulsive lifestyle choices. Traditional treatments such as CBT require the patient to utilise their own degree of cognitive abilities (willpower), which may not be functioning well due to poor health. A direct line to brain areas such as the amygdala could circumnavigate the necessity to use slower cortical areas when reprogramming the patient towards healthier decision-making. This presentation will provide suggestions for how to integrate therapy into tailored songs.
\end{abstract}

Keywords: Borderline personality disorder, memory, decision making, music therapy, CBT.

\section{Introduction}

Borderline Personality Disorder (BPD) has received the most attention from researchers due to its clinical difficulties (Paris, 2019). BPD is associated with higher rates of Cardiometabolic Syndrome (CMS), which negatively affects cognitive functioning. Cognitive function is required for traditional treatments such as DBT and CBT to be effective. Trait impulsivity can result in making poor health decisions and increased substance abuse, which negatively impact cognitive functioning and as such may decrease the effectiveness of treatments such as CBT. Listening to music has been shown to release dopamine (Koelsch et al., 2006), which is a neurotransmitter that reinforces learning by connecting reward and motivation. Providing pleasurable treatment in the form of entertainment by composing songs specifically tailored to people with BPD aims to provide constructive assistance within the music.

\section{The music}

Music can be manipulated to induce physiological changes because of a phenomenon called entrainment, whereby respiration and heart rate synchronise to rhythm (Stegemoller, 2014). It was first discovered in 1666 that two pendulum clocks set on a flexible surface would always eventually start to synchronise (Rosenblum \& Pikovsky, 2003). This phenomenon can also be observed in organisms. Autonomous oscillating systems within the human body, for example cardiac and respiratory functions, menstrual cycles, and the firing of neurons are all capable of moving around on their own or interacting with others (Trost, Labbé \& Grandjean, 2017). The term entrainment has been used to describe this process by which the autonomous oscillating systems within the body synchronise to music. A state of relaxation can be produced in the body by reducing the BPM (beats per minute) of a song to 60 (equivalent to one beat per second) which is the average healthy resting heart rate for an adult (Ellis \& Thayer, 2010). Therefore, the songs written for people with BPD aim to reach 60-80BPM to ensure listeners achieve this benefit. Fear, emotional turmoil, depression and impulsivity are traits which people with BPD exhibit. The composition process of the songs for people with BPD draws on research which indicates that mental and physical health are supported by the nervous system being in a calm state, and that music is a tool which can be utilised to reduce an organism's respiration and heart rate. 


\section{The physiology}

The vagus nerve connects the brain to the heart, and is often called the pacemaker (Cyranowski et al., 2011). Its electrical signal delivery is protected by a layer of fatty tissue, the production and strengthening of which is achieved by adapting to a stable environment. The name given to the level of protection and effectiveness of the vagal nerve is called vagal tone. Low vagal tone contributes to how childhood trauma can be associated with adverse mental and physical health in adulthood. Low vagal tone may result in many of the same illnesses which commonly result from trauma and are associated with personality disorders. Slow-paced breathing is required to practice as a technique to strengthen vagal tone which improves overall health (Lehrer \& Gevirtz, 2014). Infants who have been raised in a stable environment have learnt to breathe slowly and have good control over their Autonomic Nervous System (ANS) due to the human body's ability to match respiration and other automatic functions to other people around them. By utilising the body's natural proclivity to synchronise respiration to the beat in music, strategic compositions may strengthen vagal tone in listeners by setting the final tempo to a BPM of 60-80.

Entrainment may then serve as an important part of regulating emotions as well as supporting health (homeostasis within the organism) because of its ability to control the heart, and the heart's connection to the brain via the vagus nerve which improves cognitive functioning in preparation for any further psychological cognitive therapies. One must not separate the health of the body from the health of the mind. Instead, we should take advantage of their interconnectedness to better serve BPD sufferers. Music's connection to the amygdala is the mechanism by which the message inside a song is able to be more easily delivered to the listener.

\section{Song content}

Compositions specifically for people with BPD seek to bring awareness and understanding to sufferers about why they struggle to make healthy decisions, and then provide self-regulation advice. BPD sufferers are extremely sensitive to perceiving danger where there is not any because of their past history of trauma. Their hostile reactions in response to non-threatening situations stem from the psychological damage to their decision making processes. Humans make decisions by predicting future projections for the end result of each choice, which is calibrated based upon past experiences. For example, if you had your hand forced onto a hot stove every time you answered a maths question wrong as a child, you may feel physically nauseous at the sight of a math textbook as an adult. Your inappropriate recoiling or avoidance of equations would seem confusing to an outsider. Similarly, when soldiers return from combat they may recoil at the sound of a car exhaust pipe backfiring. When faced with a situation a healthy person may regard as normal, BPD sufferers may explode with a range of categorizable reactions due to their faulty decision-making process 'informing' their body that some form of danger is imminent when in fact it may not be (LeGris, 2018; Sobhani \& Bechara, 2011). The early childhood experiences which have shaped the BPD sufferer's decision-making processes creates a subconscious paradigm which tells them the 'wrong' results to expect as an outcome of events and choices in adult life. Therapists are recommended to use songs to bring awareness to this mechanism by which the person with BPD is triggered and then reacts inappropriately. Specific examples may be drawn from the patient's recounted experiences. Research into brain function has given way to treatments which teach self-regulation by being aware of one's physiological reactions (van der Kolk, 2014; Axmacher et al., 2010; Chiesa \& Serretti, 2011; Goodman \& Calderon, 2012). Self-regulation will be a key skill to be included in the songs. Personality disorders are an example of maladaptive strategies; adaptations to a dysfunctional early environment which became static personality traits of the individual into adulthood.

\section{Conclusion}

Patients with BPD who suffer from CMS as a result of their impulsive lifestyle decisions may benefit from therapy being delivered in the form of music which connects to areas of the brain responsible for emotional processing. This method may be beneficial for use with patients diagnosed with other Personality Disorders. Therapists with an ability to compose music can compose simple songs to act as a carrier to deliver advice to BPD patients' emotional processing centers of the brain without needing to rely on healthy cognitive processing abilities. The lyrics should raise awareness to the listener about how to make healthier decisions and how to self-regulate their behaviour and emotions. These can be personal and specific to each client depending on their life experiences which brought them in for treatment. The music must be a tempo between $60-80 \mathrm{BMP}$ so that the listener's nervous system is brought into a relaxed state. Recommendations for future research include patients taking part in the composition process. 


\section{References}

Axmacher, N., Do Lam, A. T. A., Kessler, H., Fell, J. (2010). Natural Memory Beyond the Storage Model: Repression, Trauma, and the Construction of a Personal Past. Frontiers in Human Neuroscience, 4. doi:10.3389/fnhum.2010.00211

Chiesa, A., Serretti, A. (2011). Mindfulness based cognitive therapy for psychiatric disorders: A systematic review and meta-analysis. Psychiatry Research, 187(3), 441-453. doi:10.1016/j.psychres.2010.08.011

Cyranowski, J. M., Hofkens, T. L., Swartz, H. A., Gianaros, P. J. (2011). Thinking about a close relationship differentially impacts cardiovascular stress responses among depressed and nondepressed women. Health Psychology, 30(3), 276-284. doi.org/10.1037/a0023005

Goodman, R., Calderon, A. (2012). The Use of Mindfulness in Trauma Counseling. Journal of Mental Health Counseling, 34(3), 254-268. doi:10.17744/mehc.34.3.930020422n168322

Koelsch, S., Fritz, T., v. Cramon, D. Y., Müller, K., Friederici, A. D. (2006). Investigating emotion with music: An fMRI study. Human Brain Mapping, 27(3), 239-250. doi:10.1002/hbm.20180

LeGris, J. (2018). Rapid emotional response and disadvantageous Iowa gambling task performance in women with borderline personality disorder. Borderline Personality Disorder and Emotion Dysregulation, 5(1). doi:10.1186/s40479-018-0092-x

Lehrer, P. M., Gevirtz, R. (2014). Heart rate variability biofeedback: how and why does it work? Frontiers in psychology, 5, 756. doi.org/10.3389/fpsyg.2014.00756

Paris, J. (2019). Suicidality in Borderline Personality Disorder. Medicina, 55(6), 223. doi:10.3390/medicina55060223

Rosenblum, M., Pikovsky, A. (2003). Synchronization: From pendulum clocks to chaotic lasers and chemical oscillators. Contemporary Physics, 44(5), 401-416. doi: $10.1080 / 00107510310001603129$

Sobhani, M., Bechara, A. (2011). A somatic marker perspective of immoral and corrupt behavior. Social Neuroscience, 6(5-6), 640-652. doi:10.1080/17470919.2011.605592

Stegemoller, E. L. (2014). Exploring a Neuroplasticity Model of Music Therapy. Journal of Music Therapy, 51(3), 211-227. doi:10.1093/jmt/thu023

Trost, W. J., Labbé, C., Grandjean, D. (2017). Rhythmic entrainment as a musical affect induction mechanism. Neuropsychologia, 96, 96-110. doi:10.1016/j.neuropsychologia.2017.01.004

van der Kolk, B. A. (2014). The body keeps the score: Brain, mind, and body in the healing of trauma. New York: Viking. 